\title{
Periocular rejuvenation using hyaluronic acid fillers
}

\author{
Kasra Ziai' ${ }^{1}$, Jessyka G. Lighthall ${ }^{1,2}$ \\ 'Department of Otolaryngology-Head and Neck Surgery, The Pennsylvania State University, College of Medicine, Hershey, PA \\ 17033, USA. \\ 2Facial Plastic and Reconstructive Surgery Division, Department of Otolaryngology-Head and Neck Surgery, The Pennsylvania \\ State University, College of Medicine, Hershey, PA 17033, USA.
}

Correspondence to: Dr. Jessyka G. Lighthall, Facial Plastic and Reconstructive Surgery Division, Department of OtolaryngologyHead and Neck Surgery, The Pennsylvania State University, College of Medicine, 500 University Drive, H091, Hershey, PA 17033, USA. E-mail: jlighthall@pennstatehealth.psu.edu

How to cite this article: Ziai K, Lighthall JG. Periocular rejuvenation using hyaluronic acid fillers. Plast Aesthet Res 2020;7:53. http://dx.doi.org/10.20517/2347-9264.2020.151

Received: 16 Jul 2020 First Decision: 24 Aug 2020 Revised: 27 Aug 2020 Accepted: 14 Sep 2020 Published: 12 Oct 2020

Academic Editor: Wen-Guo Cui Copy Editor: Cai-Hong Wang Production Editor: Jing Yu

\begin{abstract}
The eyes and periocular region are critical for emotive display and play a key role in social interactions. This region includes the upper and lower eyelids, brow-lid complex, and lid-cheek complex. Perturbances in this area can lead to a prematurely aged appearance and patients complain of emotive misinterpretation. It often shows the earliest signs of facial aging, leading to a tired, sad, or angry appearance. With the evolution of medical and surgical knowledge on facial aging, there has been a shift from isolated volume reducing interventions for periorbital aging to volume replacement techniques. The treatment of periocular aging is multifactorial and often includes resurfacing, chemodenervation, surgical interventions, and volumization. The minimally-invasive, office-based nature of fillers has resulted in their increased popularity and filler placement has become one of the most commonly performed cosmetic oculoplastic interventions. With a multitude of fillers emerging over the past decade or so, facial plastic surgeons have been equipped with the means to address age-related periorbital hollowing and skeletonization in an outpatient setting. An appropriate knowledge of periocular anatomy, types of fillers, proper injection technique, and management of potential complications is required for safe injection and to achieve optimal aesthetic outcomes. This paper reviews the use of hyaluronic acid fillers for periocular rejuvenation.
\end{abstract}

Keywords: Filler, hyaluronic acid, periocular, facial aging

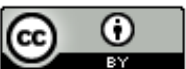

(C) The Author(s) 2020. Open Access This article is licensed under a Creative Commons Attribution 4.0 International License (https://creativecommons.org/licenses/by/4.0/), which permits unrestricted use, sharing, adaptation, distribution and reproduction in any medium or format, for any purpose, even commercially, as long as you give appropriate credit to the original author(s) and the source, provide a link to the Creative Commons license, and indicate if changes were made.

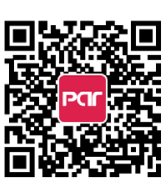




\section{INTRODUCTION}

The periocular region is essential for social interactions and conveying emotion. It is one of the first subunits of the face to show facial aging. The youthful periocular region is defined by the fullness of the periorbital area, low or no skin excess, minimal pigmentation or rhytidosis of the skin, and appropriate brow height and shape ${ }^{[1]}$. Facial aging is the result of facial fat loss, decrease in skin elasticity, deepening dynamic rhytids, static rhytids, change in bony structure, and muscular changes. With the evolution of medical and surgical knowledge over the past few decades on facial aging, we now have a better understanding of the underlying pathophysiology. This has resulted in a shift from volume reducing interventions for periorbital aging such as blepharoplasty with fat resection to volume replacement techniques $^{[2]}$. Originally, soft tissue ptosis was hypothesized to be the main underlying etiology of periocular aging. However, facial volume loss and redistribution as well as bony resorption have been proposed as contributing factors ${ }^{[2,3]}$. Since 2004 , hyaluronic acid (HA) filler has been used for periorbital rejuvenation ${ }^{[4,5]}$. The minimally-invasive, office-based nature of HA filler use without the need for general anesthesia, the low complication rate, and the lack of downtime have resulted in increased popularity, and it has replaced blepharoplasty as the most commonly performed cosmetic procedure for periocular rejuvenation ${ }^{[6]}$.

In this review, we focus on the use of HA fillers in the periocular region. However, it should be noted that the treatment of periocular aging is often multifactorial. Younger patients with early hollowing may only require HA filler for adequate rejuvenation. Older patients may benefit from multiple surgical interventions such as a brow lift, upper and/or lower blepharoplasty with or without fat transposition, or a blepharoptosis repair. Many patients also require neuromodulation for dynamic rhytids, resurfacing with laser or peels, light- or energy-based therapies, or might be better suited for surgical volumization with abdominal fat transfer alone or in combination with other treatments. Therefore, periocular rejuvenation should be individualized to patient need and surgeon experience.

\section{ANATOMY}

Knowledge of periocular anatomy as well as changes that occur during aging is critical for the appropriate treatment of patients with periocular aging and the selection of ideal candidates for HA fillers. In the evaluation of the periorbital subunit, the entire brow-lid-cheek complex should be assessed and treated ${ }^{[7]}$. The eye is roughly almond-shaped and the upper and lower eyelid margins should be at or near the superior and inferior limbus, respectively. Abnormal eyelid position or an excessively high supratarsal crease may require additional workup and treatment prior to volumization with filler. Although an in-depth discussion is beyond the scope of this paper, we briefly review the changes on each periorbital component including skin, bony structure, musculature apparatus, and fat pads.

\section{Skin}

The skin of the periorbital area and specifically the tear trough region is thin and closely attached to the underlying orbicularis oculi muscle. This results in a transparent appearance and a bluish hue at baseline related to the underlying muscular structure and vasculature ${ }^{[8]}$. Additionally, hyperpigmentation and telangiectasias may occur due to long-term sun damage. Skin aging varies among different ethnicities with different Fitzpatrick skin types. Both layers of skin, dermis and epidermis, undergo age-related changes. These changes are more pronounced in the deep layer of the dermis where the collagen fibers are strongly connected to proteoglycans. With aging, this network becomes loose and less organized, resulting in fine rhytids and crow's feet lines.

\section{Bony compartment}

The facial skeleton is believed to grow and expand with aging ${ }^{[9]}$. Recent studies have shown that certain portions of the facial bones undergo resorption with aging. The superomedial and inferolateral aspects of 


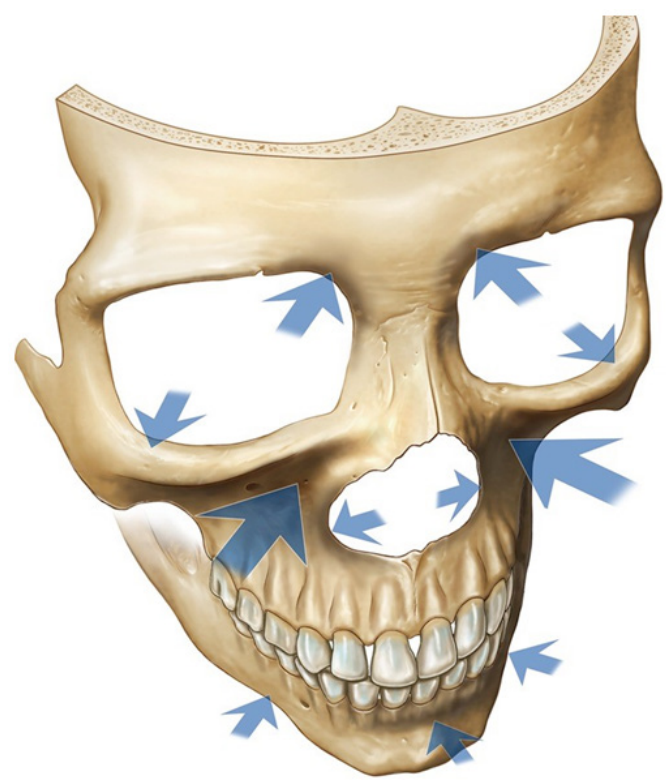

Figure 1. Bone resorption with age. In this figure, the authors demonstrate the areas of facial skeleton susceptible to bony resorption by aging. The areas with a higher degree of bony resorption are illustrated with larger arrows. Reproduced with permission from Mendelson and Wong ${ }^{[3]}$

orbital rim undergo bony resorption [Figure 1]. The inferolateral quadrant resorption of the periorbital subunit normally manifests earlier and by middle age ${ }^{[3]}$. This age-related bone loss can result in a hollow appearance of the inferolateral subunit.

In males, the supraorbital rim is more prominent and the brow is flatter and located at the level of rim, resulting in a more linear appearance ${ }^{[10]}$. In females, the aesthetic brow is arched and located above the supraorbital rim. The female brow tapers laterally ${ }^{[11]}$. In general, the highest peak of the brow should be located at the junction of the middle and lateral third at either the lateral limbus or lateral canthus.

\section{Muscles}

The periorbital muscles include the frontalis that elevates the brow and the depressor muscles which include the orbital portion of orbicularis oculi, corrugator, procerus, and depressor supercilii [Figure 2] ${ }^{[12]}$. The corrugator muscles result in vertical glabellar rhytids and the procerus results in the horizontal glabellar rhytid. These muscles are of importance when using neuromodulators in conjunction with fillers for deep static rhytids. Additionally, the orbital portion of the orbicularis oculi will contribute to the nasojugal groove due to attachments inferomedially.

\section{Fat pads}

The upper periorbital area contains the preaponeurotic fat pad, preseptal fat pad, and galea fat pad or retroorbicularis oculi fat (ROOF) pad. In periorbital aging, the preseptal and the ROOF fat descends and may lose volume, facilitating gravitational descent of the unsupported lateral brow, which causes brow ptosis and produces the appearance of a heavy lid with or without dermatochalasis. This is also attributable to the weakening of the orbital septum and subsequent pseudoherniation of the fat pads ${ }^{[13,14]}$. This will result in the deflation of the upper eyelid as well as hollowing and increased visibility of the supraorbital rim prominence. These changes produce a deep-set, hollow, and skeletonized orbit with tired, sad, or angry appearing eyes ${ }^{[1]}$.

Deep to the orbicularis is the orbital septum, which weakens with age allowing for orbital contents to bulge, producing a deepened appearance of the infraorbital hollow. Fascial extensions from the dermis to the 


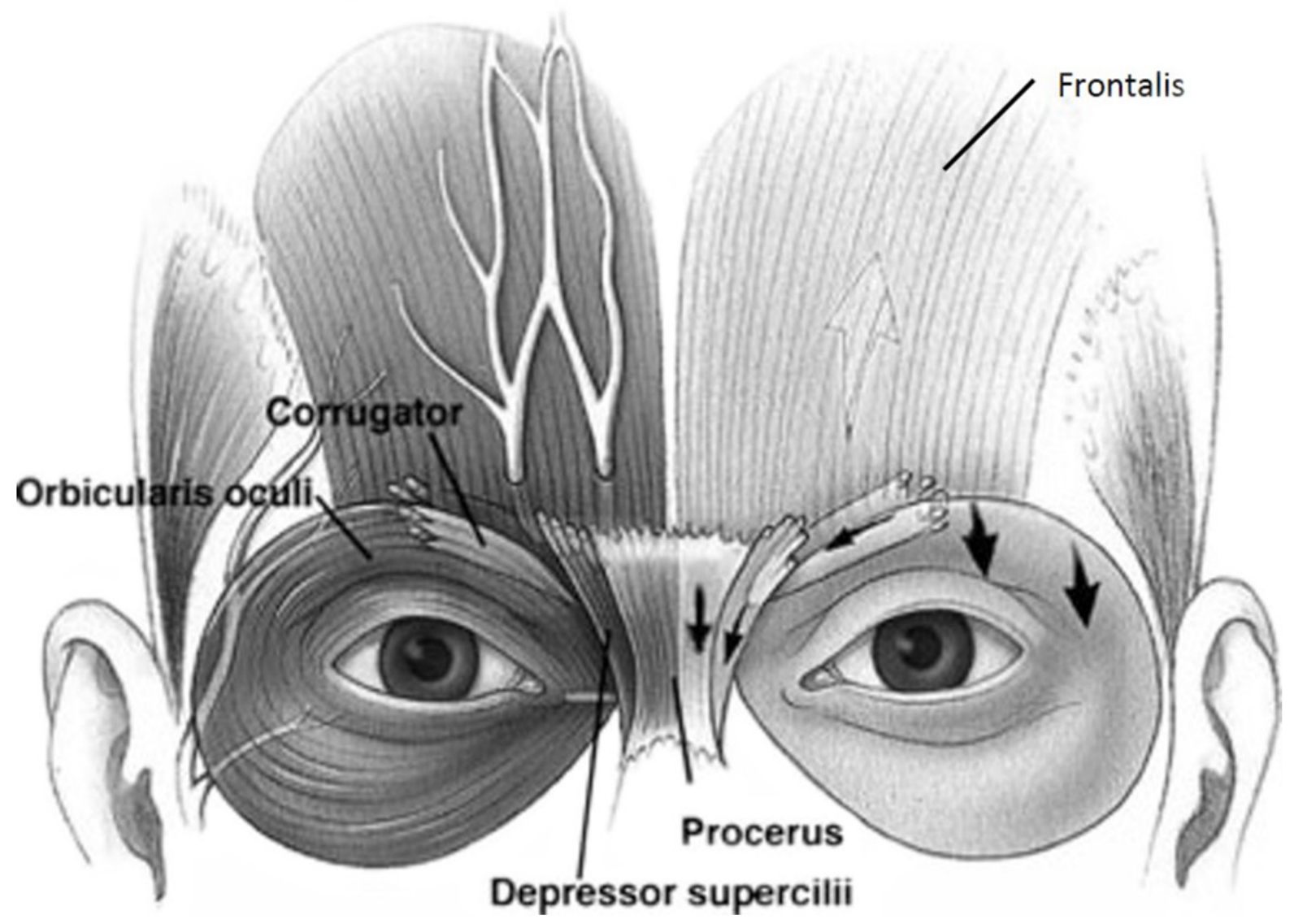

Figure 2. Periocular musculature. Reproduced with permission from Maas ${ }^{[1]}$

orbital rim (orbital retaining ligament) loosen with age, further accentuating this hollow ${ }^{[15,16]}$. These changes contribute to the classic "double convexity deformity" at the lid-cheek complex. Adding volume with HA fillers in this region can restore the youthful appearance of the periorbital area with a minimally invasive procedure. However, severe pseudoherniation of fat may limit the amount of correction obtained without surgical intervention.

\section{Periorbital blood supply}

One of the uncommon but major complications of filler injections is vascular occlusion or embolization that can result in devastating outcomes including blindness and skin necrosis. Appropriate knowledge of periorbital vascular anatomy and treatment of vascular compromise is critical for any provider injecting fillers.

The periorbital blood supply arises from both internal and external carotid arterial branches. The supratrochlear and supraorbital arteries are branches of the ophthalmic artery that arises from the internal carotid artery. The supratrochlear artery passes anteriorly through the superomedial orbit. The supraorbital artery terminal branches anastomose with the supratrochlear artery and the frontal branch of the superficial temporal artery. The angular artery is a terminal branch of the facial artery that arises from the external carotid artery. The angular artery travels along the medial angle of the orbit. Another branch of the external carotid artery is the infraorbital artery, which travels along the orbital floor and exits from the infraorbital foramen below the orbital $\operatorname{rim}^{[17]}$. The periorbital veins drain into the internal and external jugular veins as well as the cavernous sinus via the post-tarsal venous system. 


\section{PATIENT EVALUATION}

Prior to any filler use, a thorough history of any prior aesthetic procedures including prior filler or neuromodulator use, surgical interventions, lasers, or light therapy should be clarified. If a patient has a history of prior filler use, the amount and dosage (if known), as well as the type of material used should be discussed. This discussion should also include any complications or dissatisfactions. Any functional concerns such as visual field obstruction or poor eye closure should be assessed.

Additionally, a basic medical history should be obtained with specific attention to a history of inflammatory, autoimmune, or infectious disease. An ocular history including vision complaints, eye surgeries, or dry eye symptoms should be assessed. Medications should be reviewed specifically for ocular lubricant use and any prescription or over the counter anticoagulants. It is pertinent to review and clarify any allergies to medications including local anesthetics, as many HA fillers include lidocaine and topical anesthetics are commonly used prior to filler injections. Patients should also be asked about pregnancy or breastfeeding status.

A physical exam should be performed with specific attention to volume loss, volume descent, periorbital hollowing and skeletonization, pseudoherniation of fat, skin quality, Fitzpatrick skin type, dynamic and static rhytids, and globe and brow position.

A discussion with the patient regarding realistic goals and expectations is important to produce a satisfactory result. For example, a patient with significant pseudofat herniation, severe midfacial volume descent, and a deep infraorbital groove may benefit more from surgical intervention if their goals are to have a youthful, smooth lid-cheek junction. However, this patient may not be a surgical candidate or just want a mild effacement of the groove without dramatic results. As many patients are not aware of the proper treatment for their condition, it is the physician's responsibility to provide them with appropriate insight.

Ultimately, a detailed conversation of the potential risks, benefits, and complications, temporary nature of HA fillers, and alternatives must be performed prior to treatment, and obtaining written consent is recommended.

\section{Indications}

Periocular structural changes with aging are categorized into dynamic and static changes. The dynamic component is related to muscular activities and changes in resting muscle tone. The static component is defined as the changes related to volume loss as well as bony and fat pad changes ${ }^{[14]}$. Fillers are ideal to address the static component such as deep furrows and lines as well as age-related volume loss and skeletonization. Filling material can also be used for postsurgical or posttraumatic deformities in the periocular region.

\section{Contraindications}

Any active skin or localized infection at the site of injection is an absolute contraindication to filler injection. Other absolute contraindications include hypersensitivity/allergy to filler components, including lidocaine, and active collagenoses, such as mixed connective tissue disease, active systemic lupus, and active morphea [Table 1] ${ }^{[18]}$. Although active anticoagulation use is not an absolute contraindication, patients should be asked to stop the anticoagulation medication one week prior to the injection if possible. If they are not able to suspend their therapy, they should be counseled on the possibility of extensive bruising.

\section{HA FILLERS}

Prior to the availability of HA Fillers, autologous fat was predominantly used to restore volume around the eye. Autologous fat requires a donor site, is more invasive, and requires more downtime with a higher 
Table 1. Contraindications to filler use ${ }^{[18]}$

\begin{tabular}{lc}
\hline Condition & Contraindication \\
\hline Active skin infection such impetigo or herpes simplex & $\times$ \\
Hypersensitivity to filler components & $\times$ \\
Active collagenoses conditions & $\times$ \\
Graft versus host disease & $x$ \\
Systemic bacterial infections such Tuberculosis & $x$ \\
Pregnancy and breastfeeding & $x$ \\
Coagulation disorders such as hemophilia & \\
Active anticoagulant medication use & $\times$ \\
Marfan Syndrome, Ehler-Danlos Syndrome & $\times$ \\
HIV & \\
Transplant patients & $\times$ \\
\hline
\end{tabular}

rate of complications ${ }^{[19,20]}$. Although other fillers had been available, in 2003, the first HA filler (Restylane; Galderma) was approved by the FDA, and, in 2004, it was used for periorbital rejuvenation ${ }^{[20]}$. Since the introduction of the first filler, a multitude of synthetic fillers have been introduced to the market. Volume replacement occurs primarily through the hydrophilic biomaterial properties of hyaluronic acids that act as a spacer within the tissue planes. Synthetic fillers such as calcium hydroxyapatite (CHA: Radiesse; MerzAesthetics), polymethyl methacrylate (PMMA; Artefill; Suneva Medical), and poly-l-lactic acid (PLLA: Sculptra Aesthetic) have additional biostimulatory effect in addition to spacer effect ${ }^{[2]}$.

Currently, there are a multitude of synthetic HA fillers available in the market and each has different properties and characteristics [Table 2]. These properties include: concentration of the HA, particle size, viscous modulus (G'), elastic modulus (G'), and the percentage of cross-linking. Elastic modulus (G') is essentially a measure of the fillers' firmness, with a higher G' indicating a stiffer product and a lower G' indicating a softer filler ${ }^{[22]}$. Other factors that may affect physician's and patient's decisions on choosing the type of filler are needle size, cost, duration, and presence of lidocaine in the filler. These differences make each HA filler unique and ideal for a specific region of the face that is aligned with the patient's goal. In general, fillers with smaller particle size, lower concentration, and lower G' are more appropriate for fine lines and wrinkles that require more superficial injection, while fillers with higher particle size and elastic modulus are more appropriate for deeper injections. A detailed discussion of each filler and its properties are beyond the scope of this article. However, when selecting a filler for periorbital volumization, one should consider these properties. A patient with skeletonizing and significant hollowness over the infraorbital region and rim requires deeper injection compared to a patient who wants to address crow's feet, glabellar, or fine periorbital rhytids. Fillers with low affinity to water such as Restylane and lower G' such as Belotero, Voluma, and Volbella are preferred in periocular rejuvenation.

\section{PREPARATION AND TECHNIQUE}

A topical anesthetic such as a combination of $1 \%$ lidocaine/4\% tetracaine cream is applied widely over and around the planned site of injection. This cream should be left in place for at least 20 min for adequate anesthesia. In our experience, this will provide satisfactory anesthesia without the need for injectable local anesthetics. Prior to injection, skin is cleansed with alcohol and/or 0.5\% chlorhexidine based on surgeon preference with care to avoid the eye. The sites of injection may be marked if desired. HA filler may be injected with either a needle or a cannula, based on physician preference, although many feel that there is less risk of vascular occlusion when injecting with a blunt-tipped cannula. The senior author prefers the use of cannulas in the periorbital region. Key factors to consider while injecting are the depth of the desired injection, the speed of injection, the location of the periocular vasculature, and aspiration prior to injection. 
Table 2. Commonly used hyaluronic acid fillers and their properties with recommended needle size for administration

\begin{tabular}{|c|c|c|c|c|c|}
\hline Filler & Manufacturer & HA composition $(\mathrm{mg} / \mathrm{mL})$ & Presence of lidocaine & Needle size (Gauge) & $\mathbf{G}^{\prime}(\mathbf{P G})$ \\
\hline Restylane-L & Galderma & 20 & + & $29-30$ & 864 \\
\hline Restylane Lyft (Perlane) & Galderma & 24 & - & $27-29$ & 977 \\
\hline Restylane Silk & Galderma & 20 & + & 30 & - \\
\hline Belotero & Merz North America & 22.5 & - & 30 & 128 \\
\hline Juvederm Ultra XC & Allergan & 20 & + & 27 & - \\
\hline Juvederm Ultra Plus XC & Allergan & 24 & + & 27 & 207 \\
\hline Juvederm Voluma XC & Allergan & 20 & + & 27 & 398 \\
\hline Juvederm Vollure XC & Allergan & 17.5 & + & 30 & - \\
\hline Juvederm Volbella XC & Allergan & 15 & + & 30 & 240 \\
\hline Versa & Revanesse & $22-28$ & + & - & - \\
\hline
\end{tabular}

This is not an exhaustive list of all HA fillers. The G' properties and HA composition are adapted from Greene and Sidle ${ }^{[22]}$ and Cho et al..$^{[32]}$. HA: hyaluronic acid; G': elastic modulus
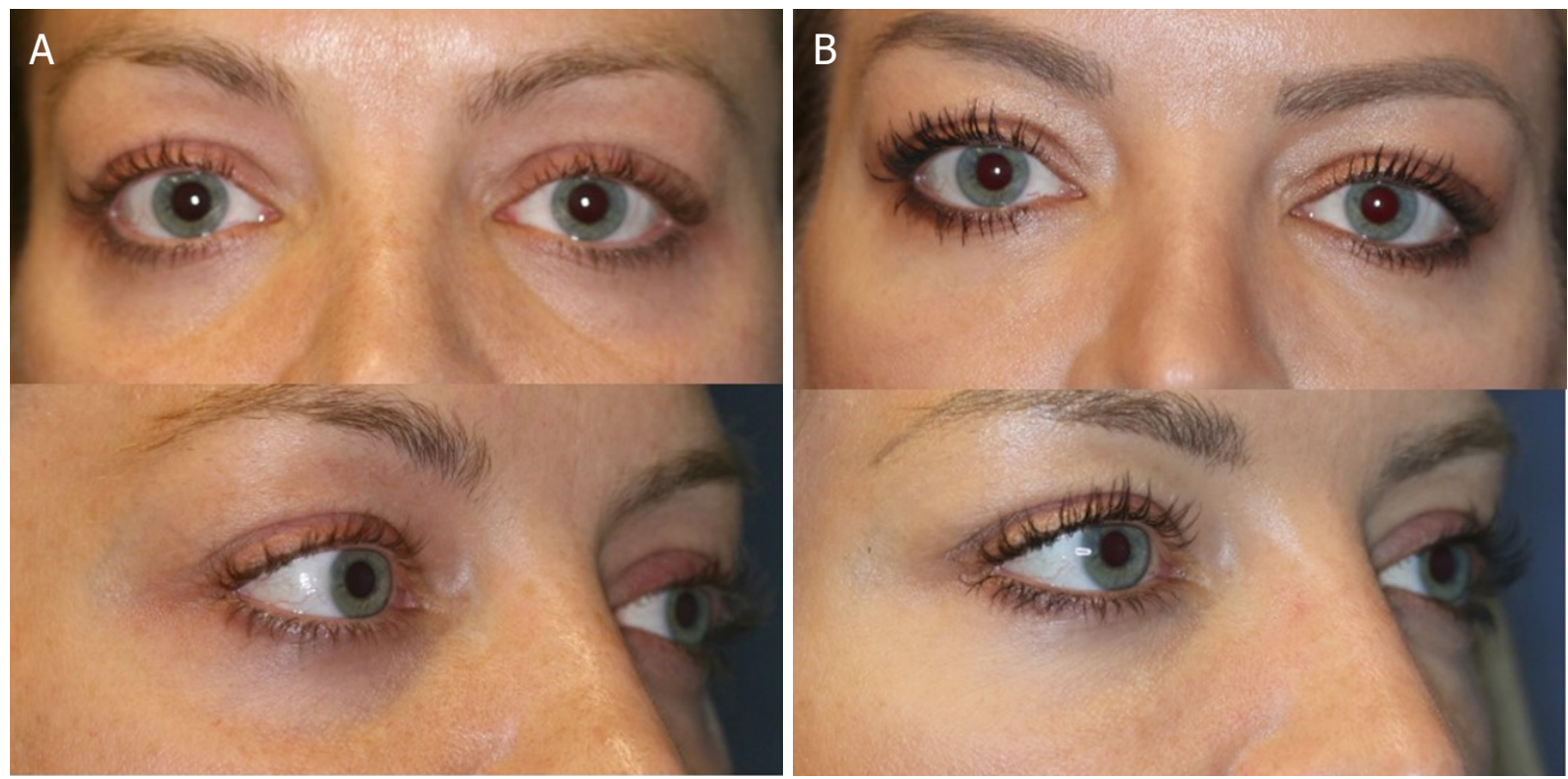

Figure 3. Tear trough deformity injections: (A) pre-injection; and (B) four months post-injection of $0.5 \mathrm{~mL}$ each side into the infraorbital hollows and $0.1 \mathrm{~mL}$ to the glabellar rhytids with Restylane- $\mathrm{L}$

\section{Tear trough}

Different injection techniques exist. If using a cannula, an entry hole is made with a 21- or 25-gauge needle depending on the cannula size below the infraorbital hollow. A cannula is then passed to the orbital retaining ligament and dry tunnels may be created along the length of the hollow followed by injection while withdrawing the cannula until the desired contour is obtained. If using a needle, one typically enters superior to the upper nasolabial fold, again passing superiorly along the orbital retaining ligament until meeting the resistance of the orbital rim, and injecting in a supraperiosteal plan. In younger patients or those with shallow folds, as little as $0.2-0.3 \mathrm{~mL}$ of filler per side is adequate for restoring volume. Caution should be made not to enter the orbit, injure the globe, and not to inject too superficial as this will result in increased bruising, a grayish or bluish discoloration, or irregularity. The area should be massaged after the injection to achieve a smooth contour [Figure 3]. If necessary to create a more rejuvenated appearance, injections into the malar eminence with a high G' filler may be combined with tear trough injections to create a more natural lid-cheek junction and restore the Ogee line [Figure 4]. These injections should be supraperiosteal and are typically injected in aliquots then massaged to provide a smooth contour. Examples of HA fillers commonly used in the malar region include Juvederm Voluma (Allergan) or Restyalne Lyft (Galderma). 

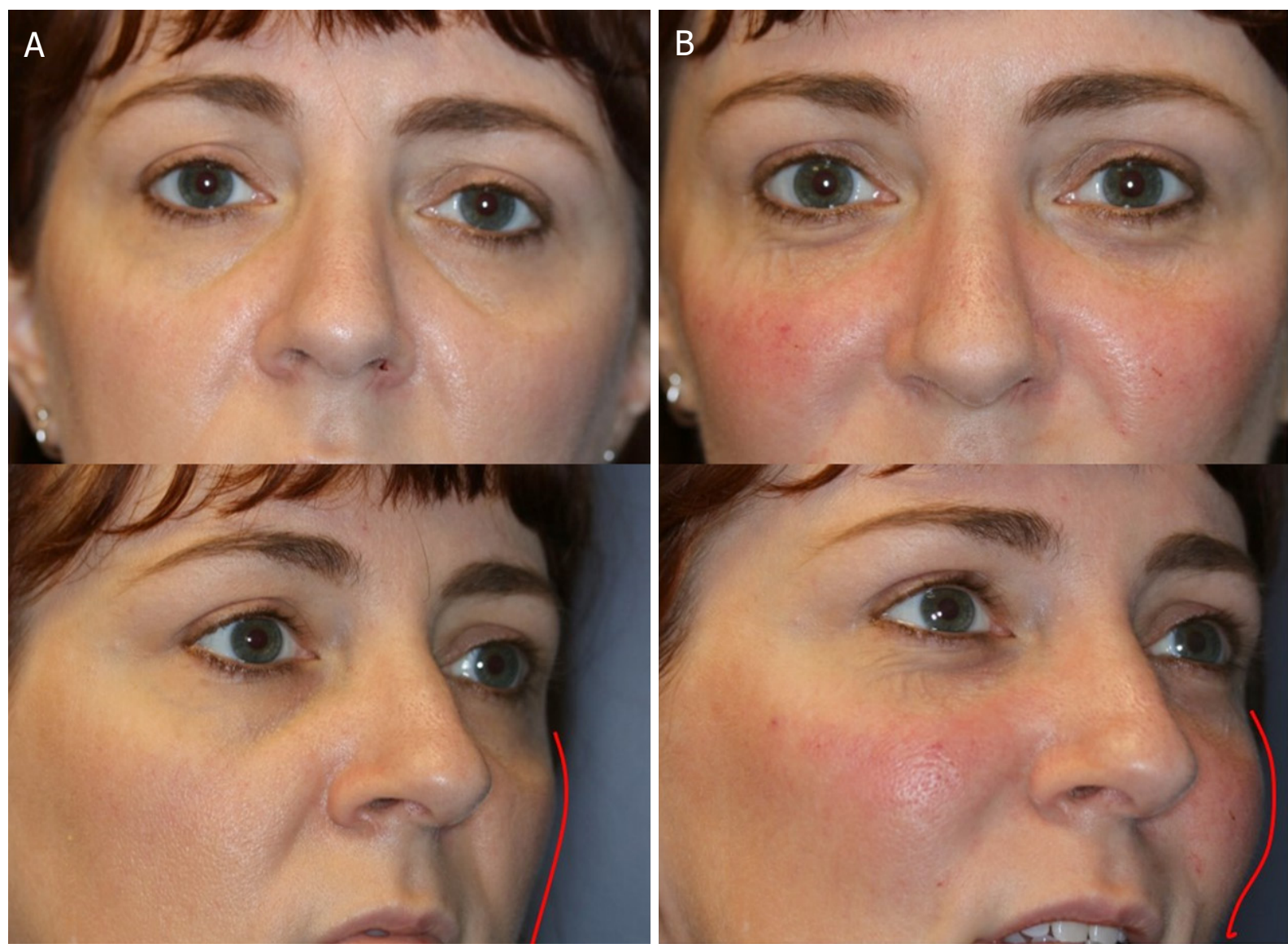

Figure 4. Hyaluronic acid injection to the tear trough and malar eminences: $(A)$ pre-injection; and $(B)$ post-injection to the infraorbital hollows with $1 \mathrm{~mL}$ of Volbella XC on each side as well as $1 \mathrm{~mL}$ of Voluma XC to each malar and infraorbital region

\section{Superior sulcus}

The hollowing of the superior sulcus with deep supratarsal crease can be addressed by injection into the sub-orbicularis plane. A low G' filler such as Belotero (Merz North America), Restylane Silk (Galderma), or Volbella (Allergan) should be utilized. Prior to injection, the superior orbital rim and location of the supraorbital notch should be identified to avoid injury to the supraorbital nerve or vascular compromise. The needle or cannula should enter along the superior orbital rim at a $30^{\circ}$ angle. After the needle has reached the bone, it should be slightly withdrawn to the preperiosteal space. An injection of as little as $0.1 \mathrm{~mL}$ on each side may be sufficient for mild cases and additional filler may be incrementally injected for more severe cases [Figure 5].

\section{Brow/temple complex}

A similar injection technique may be used to subtly elevate the brow by injecting preperiosteally just below the brow along the supraorbital rim laterally. This is also a useful technique to decrease a skeletonized or hollowed appearance with a visible rim. This injection is often performed in conjunction with superior sulcus injections. In such cases, to optimize the appearance of the periorbital region, supraperiosteal injections along the medial temple at the lateral brow may also help produce a more youthful appearance and provide subtle but powerful volumization. A high G' filler should be used in the temple to restore volume loss.

\section{Crow's feet and glabellar lines}

In many instances, the combination of neuromodulators and fillers provide a superior outcome in addressing the crow's feet, glabella, and fine periorbital rhytids. Very small aliquots of intradermal filler 


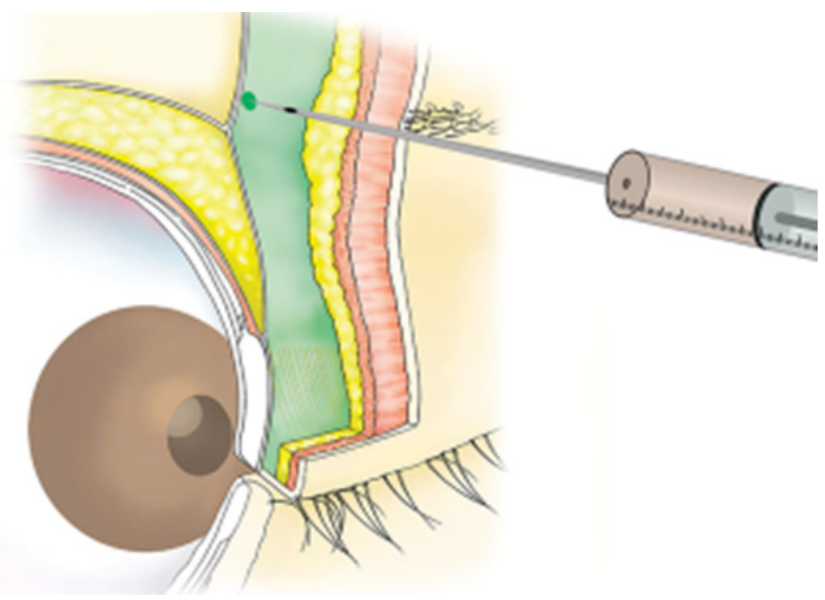

Figure 5. Hyaluronic acid injection to the superior sulcus. The needle or cannula should enter along the superior orbital rim at a $30^{\circ}$ angle. After the needle has reached the bone, it should be slightly withdrawn to the preperiosteal space to inject into the suborbicularis plane. Adapted with permission from Looi et al..$^{[31]}$
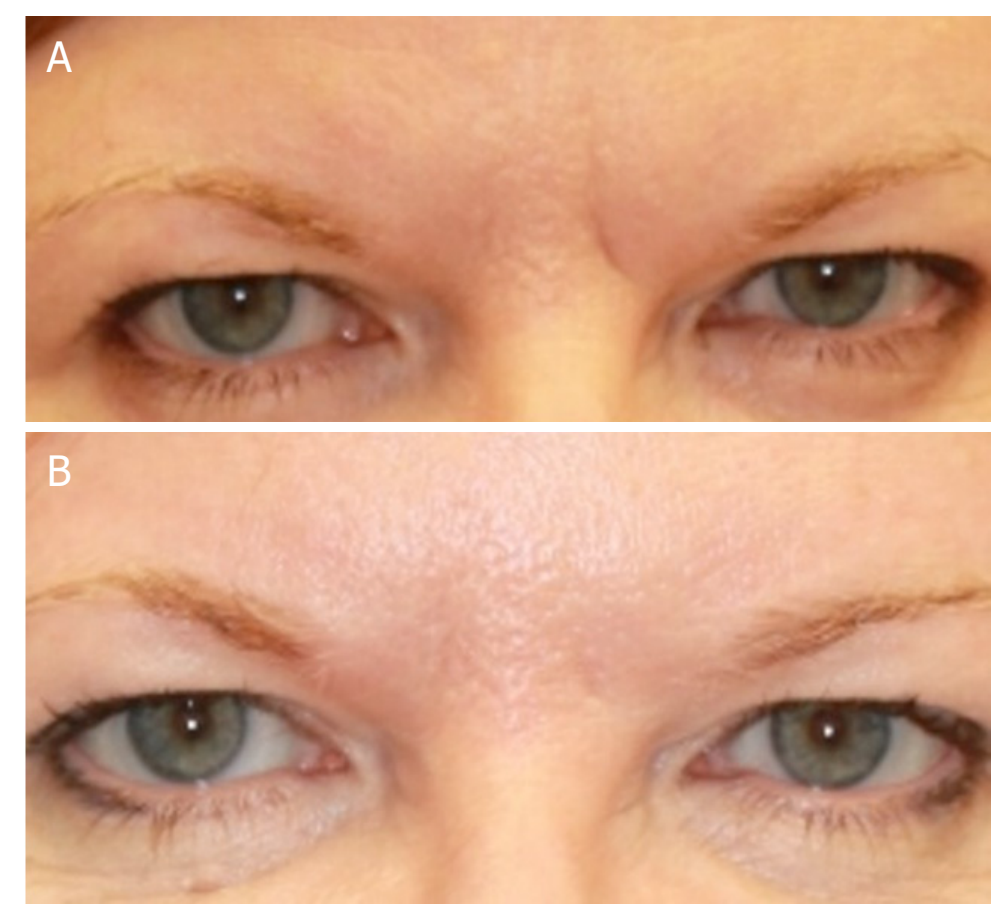

Figure 6. Combination of hyaluronic acid injection into the glabellar rhytids with chemodenervation: (A) pre-injection; and (B) two months after the glabellar injection of $0.1 \mathrm{~mL}$ on the right and $0.2 \mathrm{~mL}$ on the left of Restylane- $\mathrm{L}$

with a low G' are typically required to efface these lines. The senior author prefers using a 30-gauge needle with bevel down for these injections. The filler should be injected in the subcutaneous plane and immediately massaged after injection to achieve an even contour and avoid early-onset nodules or visibility [Figure 6].

\section{COMPLICATIONS}

\section{Vascular occlusion}

Vascular occlusion is one of the most devastating complications of filler injections. In the periocular area, this can result in intra-arterial embolization or vascular compression and subsequent vision loss or skin 
necrosis. The rate of vascular occlusion and subsequent ischemic complications has been reported up to 3 in every 1,000 injections ${ }^{[23]}$. Ophthalmic artery occlusion can result from intra-arterial injection in the periorbital region due to the multiple anastomosis between the ophthalmic artery and the dorsal nasal, supratrochlear, and supraorbital arteries with possible blindness ${ }^{[24]}$. A patient presenting with increased pain at the site of injection, visual loss, or visible signs of skin ischemia should undergo emergent evaluation for possible vascular occlusion.

Knowledge of appropriate techniques and preventive measures is important to decrease the risk of vascular complications. Aspiration prior to injection is extremely important to prevent inadvertent injection into the vessel lumen. Slow and steady injection has been shown to decrease the risk of vascular complications. It is also important to use an appropriate sized needle for injection. When the needle's gauge is too small, the risk of arterial wall damage and cannulation is higher. Consideration should be taken for the use of cannulas to decrease this risk. If severe pain occurs during injection, the procedure needs to be stopped immediately and the patient should be evaluated for possible vascular complication.

One of the advantages of HA fillers is the ability to provide reversal when complications such as skin ischemia, nodule formation, Tyndall effect, or subpar aesthetic outcomes occur by injection of hyaluronidase in a timely fashion. Although uncommon, if arterial embolization or vascular occlusion is suspected, immediate intervention is required. Treatments discussed in the literature are heat, topical nitroglycerin, hyaluronidase injection in the affected region, massage, and hyperbaric oxygen ${ }^{[23]}$. For any vision loss, immediate retrobulbar hyaluronidase injection is recommended. However, this treatment modality remains controversial. In a single case report by Chestnut ${ }^{[25]}$, injection of 450 units of hyaluronidase in the retrobulbar area was reported to reverse the vision loss caused by periorbital HA filler injection. However, the nature of the vision loss was not documented.

In a study by Lee et al ${ }^{[26]}$, the effectiveness of retrobulbar hyaluronidase was investigated in rabbit models for possible reversal of HA filler related blindness. The authors found that in three out of four experimented eyes, the retinal perfusion was increased with normal electroretinography after retrobulbar hyaluronidase injection.

In another study by Paap et al. ${ }^{[27]}$ the effectiveness of retrobulbar hyaluronidase was investigated in a human cadaver model. In this study, the authors demomstrated that retrobulbar hyaluronidase injection is unlikely to be effective in management of HA filler induced central retinal artery occlusion and blindness due to its inability to cross dural sheet of the optic nerve. A review of the medical literature by Paap et al ${ }^{[28]}$ regarding the efficacy of retrobulbar hyaluronidase as a treatment for filler related blindness concluded that to date this is an unproven therapy and largely unsuccessful.

Although this treatment modality has not been proven successful and further studies are needed to evaluate the effectiveness, an immediate retrobulbar hyaluronidase injection may be performed; however, patients must be informed that there is no current evidence-based, effective, and consistent treatment to reverse this devastating complication.

\section{Bruising, redness, and swelling}

Redness, bruising, and swelling are the most common side effects related to filler injection. The redness and bruising usually happen immediately with periorbital injections caused by direct puncture of blood vessels upon entry of the needle. It has been proposed that higher gauge needles can result in less bruising and redness due to the lower risk of damage to blood vessel walls ${ }^{[24]}$. The use of a cannula may also cause less bruising due to fewer injection holes required and the blunt tip of the cannula. All patients should be advised to stop any anticoagulant medications, if possible, one week prior to treatment to decrease the 


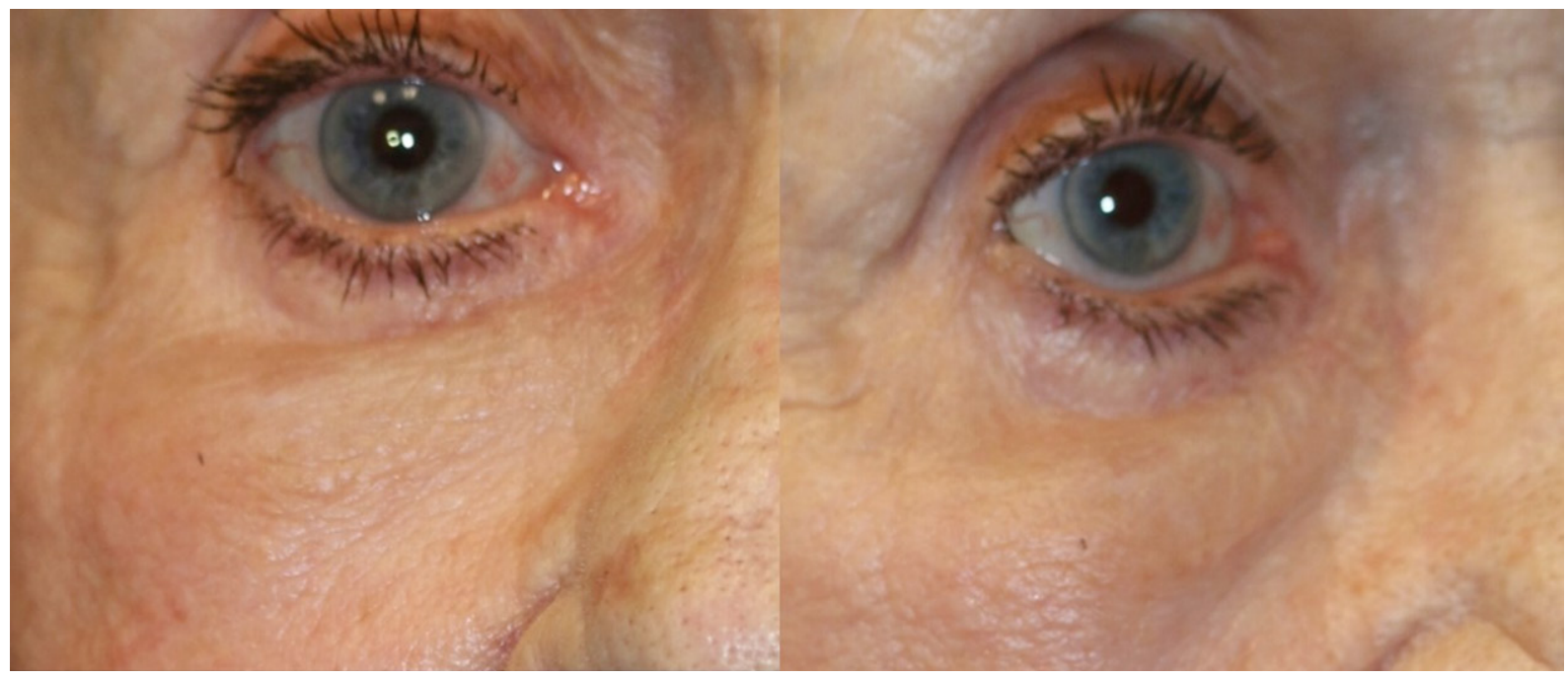

Figure 7. Antero-posterior and oblique view of a 76-year-old female who presented for a blepharoplasty consult. She had undergone blepharoplasty and hyaluronic acid injection to the tear trough by another provider years prior and noted a chronic swelling and discoloration in the under-eye. The exam showed a mild chronic inflammatory reaction with palpable curvilinear nodule with overlying edema. Small aliquots of hyaluronidase were injected with the resolution of the inflammation

risk of bruising and hematoma formation. Occasionally, patients develop more prominence of their native vessels after injection that may benefit from laser treatment.

\section{Tyndall effect (blue-grey dyschromia)}

Superficial injections of fillers can result in bluish hue caused by light passing through colloid. Injections into the tear trough are more prone to Tyndall effect due to the thin, almost translucent nature of the skin at this location. This blue-grey discoloration can happen weeks to months after HA injection into the periorbital region and may persist long term ${ }^{[29]}$. To decrease the risk of Tyndall effect, the needle or cannula bevel should be pointed inferiorly at the time of injection and the product may be injected in a supraperiosteal plane. Makeup and phototherapy can be used for the management of blue-grey dyschromia as well as hyaluronidase injections.

\section{Nodules}

Another side effect of fillers is delayed-onset nodules. These nodules are thought to be caused by local or systemic infections or inflammatory reactions and the incidence has been reported to be higher during winter months, which is thought to be related to a higher incidence of upper respiratory infections ${ }^{[30]}$. These nodules can appear years after injections and have also been postulated to be due to biofilm formation. These may appear in the tear trough region as a long, curvilinear swelling or irregularity along the infraorbital hollow that is mobile and may have surrounding edema [Figure 7]. Treatment with a prolonged course of empirical antibiotics such as macrolides has been recommended for their anti-inflammatory properties in addition to the antimicrobial effect. Injection of steroids into the nodule, a short-course of systematic steroids, and hyaluronidase are other potential options for management ${ }^{[24]}$.

\section{CONCLUSION}

HA fillers provide an excellent option for a minimally invasive approach to periocular rejuvenation. With the emergence of a multitude of HA fillers over the past decade, facial and oculoplastic surgery has been equipped with the means to address age-related periorbital hollowing and skeletonization in an outpatient setting. Appropriate knowledge of periocular region anatomy, characteristics of HA fillers, proper injection technique, and management of complications is required for safe injection and to achieve optimal aesthetic outcomes. 


\section{DECLARATIONS}

\section{Authors' contributions}

Study concept and design; drafting, critical revision of the manuscript for important intellectual content; collected the data; administrative, technical, or material support: Ziai K, Lighthall JG

Study supervision: Lighthall JG

\section{Availability of data and materials}

Not applicable.

\section{Financial support and sponsorship}

None.

\section{Conflicts of interest}

All authors declared that there are no conflicts of interest.

\section{Ethical approval and consent to participate}

Not applicable.

\section{Consent for publication}

The informed consent was obtained for publication of patient images used in this manuscript.

\section{Copyright}

(C) The Author(s) 2020.

\section{REFERENCES}

1. Liew S, Nguyen DQ. Nonsurgical volumetric upper periorbital rejuvenation: a plastic surgeon's perspective. Aesthetic Plast Surg 2011;35:319-25.

2. De Biasio F, Miotti G, Zingaretti N, Castriotta L, Parodi PC. Study on the aging dynamics of the periorbital region: from observation to knowledge of physiopathology. Ophthalmic Plast Reconstr Surg 2019;35:333-41.

3. Mendelson $\mathrm{B}$, Wong $\mathrm{CH}$. Changes in the facial skeleton with aging: implications and clinical applications in facial rejuvenation. Aesthetic Plast Surg 2012;36:753-60.

4. Goldberg RA, Fiaschetti D. Filling the periorbital hollows with hyaluronic acid gel: initial experience with 244 injections. Ophthalmic Plast Reconstr Surg 2006;22:335-41; discussion 341-3.

5. Kane MA. Treatment of tear trough deformity and lower lid bowing with injectable hyaluronic acid. Aesthetic Plast Surg 2005;29:363-7.

6. Mustak H, Fiaschetti D, Goldberg RA. Filling the periorbital hollows with hyaluronic acid gel: Long-term review of outcomes and complications. J Cosmet Dermatol 2018;17:611-6.

7. Lighthall JG. Rejuvenation of the upper face and brow: neuromodulators and fillers. Facial Plast Surg 2018;34:119-27.

8. Cotofana S, Fratila AA, Schenck TL, Redka-Swoboda W, Zilinsky I, et al. The anatomy of the aging face: a review. Facial Plast Surg 2016;32:253-60

9. Bartlett SP, Grossman R, Whitaker LA. Age-related changes of the craniofacial skeleton: an anthropometric and histologic analysis. Plast Reconstr Surg 1992;90:592-600.

10. Clevens RA. Rejuvenation of the male brow. Facial Plast Surg Clin North Am 2008;16:299-312, vi.

11. Maas CS. Botulinum neurotoxins and injectable fillers: minimally invasive management of the aging upper face. Otolaryngol Clin North Am 2007;40:283-90.

12. Briceño CA, Zhang-Nunes SX, Massry GG. Minimally invasive options for the brow and upper lid. Facial Plast Surg Clin North Am 2015;23:153-66.

13. Knize DM. An anatomically based study of the mechanism of eyebrow ptosis. Plast Reconstr Surg 1996;97:1321-33.

14. Kashkouli MB, Abdolalizadeh P, Abolfathzadeh N, Sianati H, Sharepour M, et al. Periorbital facial rejuvenation; applied anatomy and pre-operative assessment. J Curr Ophthalmol 2017;29:154-68.

15. Mendelson BC, Muzaffar AR, Adams WP Jr. Surgical anatomy of the midcheek and malar mounds. Plast Reconstr Surg 2002;110:88596; discussion 897-911.

16. Moon HS, Ahn B, Lee JH, Rah DK, Park TH. Rejuvenation of the deep superior sulcus in the eyelid. J Cosmet Dermatol 2016;15:458-68.

17. Sand JP, Zhu BZ, Desai SC. Surgical anatomy of the eyelids. Facial Plast Surg Clin North Am 2016;24:89-95.

18. De Boulle K, Heydenrych I. Patient factors influencing dermal filler complications: prevention, assessment, and treatment. Clin Cosmet 
Investig Dermatol 2015;8:205-14.

19. Lee S, Yen MT. Nonsurgical rejuvenation of the eyelids with hyaluronic acid gel injections. Semin Plast Surg 2017;31:17-21.

20. Attenello NH, Maas CS. Injectable fillers: review of material and properties. Facial Plast Surg 2015;31:29-34.

21. Lee JC, Lorenc ZP. Synthetic fillers for facial rejuvenation. Clin Plast Surg 2016;43:497-503.

22. Greene JJ, Sidle DM. The hyaluronic acid fillers: current understanding of the tissue device interface. Facial Plast Surg Clin North Am 2015;23:423-32.

23. Rzany B, DeLorenzi C. Understanding, avoiding, and managing severe filler complications. Plast Reconstr Surg 2015;136:196-203S.

24. Murthy R, Roos JCP, Goldberg RA. Periocular hyaluronic acid fillers: applications, implications, complications. Curr Opin Ophthalmol 2019;30:395-400

25. Chesnut C. Restoration of visual loss with retrobulbar hyaluronidase injection after hyaluronic acid filler. Dermatol Surg 2018;44:435-7.

26. Lee W, Oh W, Ko HS, Lee SY, Kim KW, et al. Effectiveness of retrobulbar hyaluronidase injection in an iatrogenic blindness rabbit model using hyaluronic acid filler injection. Plast Reconstr Surg 2019;144:137-43.

27. Paap MK, Milman T, Ugradar S, Silkiss RZ. Assessing retrobulbar hyaluronidase as a treatment for filler-induced blindness in a cadaver model. Plast Reconstr Surg 2019;144:315-20.

28. Paap MK, Milman T, Ugradar S, Goldberg R, Silkiss RZ. Examining the role of retrobulbar hyaluronidase in reversing filler-induced blindness: a systematic review. Ophthalmic Plast Reconstr Surg 2020;36:231-8.

29. Rootman DB, Lin JL, Goldberg R. Does the Tyndall effect describe the blue hue periodically observed in subdermal hyaluronic acid gel placement? Ophthalmic Plast Reconstr Surg 2014;30:524-7.

30. Beleznay K, Carruthers JD, Carruthers A, Mummert ME, Humphrey S. Delayed-onset nodules secondary to a smooth cohesive $20 \mathrm{mg} /$ mL hyaluronic acid filler: cause and management. Dermatol Surg 2015;41:929-39.

31. Looi AL, Yong KL. "Walk the Rim, Feel the Bone" technique in superior sulcus filling. Plast Reconstr Surg Glob Open 2015;3:e592.

32. Cho SY, Park JW, An H, Ko HJ, Kim H, et al. Physical properties of a novel small-particle hyaluronic acid filler: In vitro, in vivo, and clinical studies. J Cosmet Dermatol 2018;17:347-54. 